Tropical Journal of Pharmaceutical Research February 2018; 17 (2): 225-232

ISSN: $1596-5996$ (print); 1596-9827 (electronic)

(c) Pharmacotherapy Group, Faculty of Pharmacy, University of Benin, Benin City, 300001 Nigeria.

\title{
Glechoma longituba (Lamiaceae) alleviates apoptosis in calcium oxalate-induced oxidative stress in kidney proximal tubule epithelial cell line, HK-2
}

\author{
Sheng-xing Wang ${ }^{1 *}$, Pei-yu Liang ${ }^{1}$, Yi-nan Han ${ }^{2}$, Bang-fen Zhou ${ }^{1}$, Jin-huo Chen ${ }^{1}$ \\ ${ }^{1}$ Department of Urology, ${ }^{2}$ Operation Room, The Affiliated Hospital of Hainan Medical University, Hainan, 570102, China
}

*For correspondence: Email: wangshx163@163.com

Sent for review: 15 November 2017

Revised accepted: 26 January 2018

\begin{abstract}
Purpose: To investigate the effects of Glechoma longituba on calcium oxalate ( $\mathrm{CaOx}$ )-induced stress in $H K-2$ cells as a possible treatment strategy for nephrolithiasis (kidney stones).

Methods: Human kidney HK-2 cells were treated with $\mathrm{CaOx}$ and Glechoma longituba at different concentrations. The levels of reactive oxygen species (ROS), lactate dehydrogenase (LDH), and malondialdehyde (MDA) were measured. Cell apoptosis and viability were assessed by flow cytometry and 3-(4,5-dimethylthiazol-2-yl)-2,5-diphenyltetrazolium bromide (MTT) assays, respectively, while apoptosis-related proteins were determined using western blotting. The levels of the nuclear factorerythroid 2-related factor 2 (Nrf2), heme oxygenase-1 (HO-1), and NADPH-quinone-oxidoreductase 1 (NQO-1) genes were evaluated using quantitative real-time polymerase chain reaction (qRT-PCR). Using lentivirus, Nrf2 was knocked down in HK-2 cells, and this was confirmed by both qRT-PCR and western blotting. Scramble and si-Nrf2 transfected HK-2 cells were treated with $\mathrm{CaOx}$ and Glechoma longituba, and ROS levels and apoptosis were also assessed.

Results: $\mathrm{CaOx}$ significantly increased the levels of ROS, LDH and MDA, while Glechoma longituba pretreatment attenuated these elevations in a dose-dependent manner. CaOx treatment increased cell apoptosis and decreased cell viability $(p<0.05)$, while Glechoma longituba pre-treatment abolished these effects in a dose-dependent manner. Glechoma longituba pre-treatment significantly upregulated the expressions of Nrf2, HO-1 and NQO-1 $(p<0.05)$. In HK-2 cells, Si-Nrf-2 attenuated the effects of Glechoma longituba pre-treatment on cell oxidative stress and apoptosis induced by $\mathrm{CaOx}$.

Conclusion: Glechoma longituba pre-treatment attenuates cell apoptosis and oxidative stress induced by $\mathrm{CaO} x$ via Nrf2/HO-1 signalling pathway. Thus, the plant is a potential source of agents for the treatment of nephrolithiasis.
\end{abstract}

Keywords: Glechoma longituba, Nephrolithiasis, Nuclear factor-erythroid 2-related factor 2, Oxidative stress, Apoptosis

This is an Open Access article that uses a funding model which does not charge readers or their institutions for access and distributed under the terms of the Creative Commons Attribution License (http://creativecommons.org/licenses/by/4.0) and the Budapest Open Access Initiative (http://www.budapestopenaccessinitiative.org/read), which permit unrestricted use, distribution, and reproduction in any medium, provided the original work is properly credited.

Tropical Journal of Pharmaceutical Research is indexed by Science Citation Index (SciSearch), Scopus, International Pharmaceutical Abstract, Chemical Abstracts, Embase, Index Copernicus, EBSCO, African Index Medicus, JournalSeek, Journal Citation Reports/Science Edition, Directory of Open Access Journals (DOAJ), African Journal Online, Bioline International, Open-J-Gate and Pharmacy Abstracts

\section{INTRODUCTION}

Nephrolithiasis is a common kidney disease and a major cause of morbidity worldwide [1]. Over the past two decades, the prevalence of nephrolithiasis has increased by $70 \%$ among 
adults [2]. In addition, the incidence of nephrolithiasis among adolescents has also increased by $6-10 \%$ annually $[3,4]$. The increase of nephrolithiasis is a challenge to human health, and an economic burden for families and society [5]. Currently, surgery and medical treatments are the main therapies for nephrolithiasis, but the outcome remains unsatisfactory. The recurrence of nephrolithiasis is approximately $50 \%$ within 10 years of diagnosis [6]. Thus, it is important to determine the mechanism of nephrolithiasis to provide new insight for improved treatment of nephrolithiasis.

Calcium oxalate $(\mathrm{CaOx})$ stones are considered to constitute the majority of calculi in kidney stones, which are characterized by hypercalciuria, attached $\mathrm{CaOx}$ stones, and interstitial calcium phosphate deposits [7]. CaOx not only obstructs ureters, but also induces reactive oxygen species (ROS), which results in renal cellular injury and inflammation $[8,9]$. Liang et al. have reported that androgen receptor degradation enhancer, ASCJ9, inhibits the formation of $\mathrm{CaOx}$ crystals by regulating oxalate biosynthesis and oxidative stress both in vivo and in vitro [10]. In addition, erythrocyte oxidative damage contributes to the tubular damage and stone formation [11]. Recently, several herbal remedies have been used as treatments for nephrolithiasis in clinics, owing to their regulation of $\mathrm{CaOx}$ formation $[12,13]$. However, their underlying mechanisms remain unclear.

In the current study, a traditional Chinese medicinal plant, Glechoma longituba, was explored to identify its effects on oxidative damage of renal epithelial cell induced by $\mathrm{CaOx}$, to provide new insights into the treatment of nephrolithiasis.

\section{EXPERIMENTAL}

\section{Cell culture and treatment}

Human kidney epithelial cells, HK-2, and a human embryonic kidney cell line, 293T, were used in this study. The HK-2 cells were maintained in Dulbecco's Modified Eagle Medium (DMEM)/F12 medium (Gibco, Grand Island, NJ, USA) supplemented with $10 \%$ fetal bovine serum (FBS; Gibco) at $37^{\circ} \mathrm{C}$ in a humidified incubator with $5 \% \quad \mathrm{CO}_{2}$. In addition, 293T cells were maintained in DMEM medium supplemented with $10 \% \mathrm{FBS}$ at $37^{\circ} \mathrm{C}$ in a humidity incubator with $5 \% \mathrm{CO}_{2}$. When grown to $70-80 \%$ confluency, as monolayers, the HK-2 cells were seeded in a 6well plate and treated with or without $1.0,2.0$, or $4.0 \mathrm{mg} / \mathrm{mL}$ of Glechoma longituba solution. One hour later, the cells were treated with or without $67 \mu \mathrm{g} / \mathrm{cm}^{2} \mathrm{CaOx}$.

\section{Preparation of $\mathrm{CaOx}$ crystals}

To obtain $\mathrm{CaOx}$ crystals, equal volumes of 10 $\mathrm{mM} \mathrm{NaOx}$ and $\mathrm{CaCl}_{2}$ were mixed at room temperature. After crystals formed, the suspension was equilibrated at $4^{\circ} \mathrm{C}$ for 3 days. Then, the crystals were washed with doubledistilled water (DDW) twice, and dried at $60^{\circ} \mathrm{C}$. Subsequently, Fourier transform infrared (FT-IR) spectroscopy analysis was used to confirm the crystals of the formed $\mathrm{CaOx}$. After confirmation, $5 \mathrm{mg} / \mathrm{mL} \mathrm{CaOx}$ solution was prepared in sterile phosphate-buffer saline (PBS).

\section{Preparation of Glechoma longituba solution}

Glechoma longituba were purchased from Beijing Tongrentang (Beijing, China). One thousand grams of raw material was immersed in $5.0 \mathrm{~L}$ of DDW for $60 \mathrm{~min}$ and further extracted for $25 \mathrm{~min}$. The mixed materials were filtered, and the supernatant was collected. The residue was mixed with $3.0 \mathrm{~L}$ of DDW, boiled, and filtered. Then, the first and second aqueous extracts were mixed, re-filtered, concentrated, lyophilized, and stored at $-80^{\circ} \mathrm{C}$. In the current study, Glechoma longituba powder was prepared at three concentrations: $1.0,2.0$, and $4.0 \mathrm{mg} / \mathrm{mL}$, for subsequent investigations.

Assessment of reactive oxygen species (ROS), lactate dehydrogenase (LDH), and malondialdehyde (MDA)

ROS levels in HK-2 cells in 6-well plates were evaluated using the ROS-specific fluorescent dye, 2',7'-dichlorodihydrofluorescein diacetate (DCFH-DA) (Invitrogen, Carlsbad, CA, USA), according the manufacture's protocol. The LDH cytotoxicity assay detection kit (Beyotime, Haimen, China) was utilized to determine LDH levels in the cell medium according to the manufacturer's protocol. A commercial TBARS Assay Kit (Cayman Chemical Co., Ann Arbor, MI, USA) was used to detect MDA, which is a thiobarbituric acid reactive substance (TBARS) and an indicator of cell lipid peroxidation, according to the manufacturer's protocol.

\section{Assessment of apoptosis}

After treatment with Glechoma longituba, the apoptosis levels of HK-2 cells in a 6-well plate were determined using the V-FITC/PI apoptosis kit (MaiBio, Hong Kong, China) according to the manufactures protocol. The fluorescence of fluorescein isothiocyanate (FITC) and propidium 
iodide (PI) were measured using a Beckman flow cytometer and estimated using Expo32 software (Beckman Coulter, Sacramento, CA, USA).

\section{Assessments of cell viability}

Cell viability of HK-2 cells was estimated using the 3-(4,5-dimethylthiazol-2-yl)-2,5diphenyltetrazolium bromide (MTT) assay. HK-2 cells were seeded onto a 96-well plate at a density of $5.0 \times 10^{3}$ cells/well, and treated with Glechoma longituba and $\mathrm{CaOx}$ as previously mentioned. After treatment for $48 \mathrm{~h}$, cells were incubated with $2.0 \mathrm{mg} / \mathrm{mL}$ MTT solution (SigmaAldrich, St. Louis, MO, USA) for each well at $37^{\circ} \mathrm{C}$ for $4 \mathrm{~h}$. Following this, formazan crystals in cells were dissolved with dimethylsulfoxide and the optical density of the product was measured at $540 \mathrm{~nm}$ using a Varioskan ${ }^{\circledR}$ Flash Top Spectral Scanning Multimode Reader (Thermo Fisher Scientific, Waltham, MA, USA). Each sample had five replicates and each experiment was performed in triplicate.

\section{Western blotting}

After treatment, the medium was discarded and cells were lysed using RIPA lysis buffer containing a protease inhibitor cocktail (Roche, Indianapolis, IN, USA). The suspension was then ultrasonicated and centrifuged at $4^{\circ} \mathrm{C}$, at $1,000 \mathrm{~g}$, for $10 \mathrm{~min}$. The supernatant was collected, quantified using the Bradford assay, and boiled with an equal volume of loading buffer. Then, 10 $\mu \mathrm{g}$ of total protein was loaded for SDS-PAGE followed by transfer of the proteins onto a polyvinylidene difluoride (PVDF) membrane. Blots were blocked with $5 \%$ non-fat milk at room temperature for $1 \mathrm{~h}$, and then incubated with rabbit anti-human Nrf2 (1:1000, Sigma Aldrich), anti-pro-caspase-3 (1:3000; Cell Signalling Technology, Danvers, MA, USA), anti-procaspase-9 (1:1000; Cell Signalling Technology), anti-cleaved-caspase-3 (1:3000; Cell Signalling Technology), anti-cleaved-caspase-9 (1:1000; Cell Signalling Technology), anti-Bcl-2 (1:1000; Sigma-Aldrich), anti-Bax (1:2000; Sigma-Aldrich), or anti- $\beta$-actin (1:5000; Sigma-Aldrich) at $4^{\circ} \mathrm{C}$ overnight. Blots were incubated with secondary antibody at room temperature for $1 \mathrm{~h}$ and visualized using the Enhanced Chemiluminescence Detection Kit (SuperSignal West Femto, Thermo Scientific).

\section{Quantitative real-time polymerase chain reaction (qRT-PCR)}

Total RNA from HK-2 cells was extracted using TRIzol reagent (Thermo Scientific, Shanghai, China) following the manufacture's protocol.
Then, 2-3 $\mu \mathrm{g}$ of total RNA was used to synthesize cDNA using a cDNA synthesis kit (TakaRa, Tokyo, Japan). Subsequently, qRTPCR was performed using SYBR Green premix (TakaRa) on an ABI Prism ${ }^{\circledR} 7500$ instrument ( $\mathrm{ABI}$, Foster city, CA, USA). The amplification conditions were as follows: $94^{\circ} \mathrm{C}$ for $20 \mathrm{~s}, 40$ cycles at $94^{\circ} \mathrm{C}$ for $3 \mathrm{~s}$, and $55^{\circ} \mathrm{C}$ for $30 \mathrm{~s}$. The primer sequences were as follows: Nrf2, forward: 5'-TCTTGCCTCCAAAGTATGTCAA-3' and reverse: 5'-CACGGTCCACAGCTCATC-3'; HO1, forward: 5'-TGAAGGAGGCCACCAAGGAGG3' and reverse: 5'-AGAGGTCACCCAGGTA GCGGG-3'; NQO-1, forward, 5'-GCAGTGC TTTCCATCACCAC-3' and reverse: 5'-TGGAGTGTGCCCAATGCTAT-3'; and $\beta$-actin: forward: 5'-GGGGACCTGACTGACTA-3' and reverse: 5'TGAAGGTAGTTTCGTGGATGC-3'. Gene expression levels were assessed using the $2^{-\Delta \Delta C t}$ method [14] and $\beta$-actin was used as the internal control.

\section{Statistical analysis}

GraphPad Prism (version 6.0; GraphPad Software, La Jolla, CA, USA) was utilized to perform statistical analyses. Continuous variables are presented as the mean \pm standard deviation (SD). Comparisons between groups were assessed using Student's $t$-test, and a significant difference was considered when $p<$ 0.05 .

\section{RESULTS}

\section{Glechoma longituba inhibited the oxidative stress induced by $\mathrm{CaOx}$.}

ROS, LDH, and MDA levels in HK- 2 cells were significantly decreased in $\mathrm{CaOx}$-treated cells compared with the control group $(p<0.01)$. However, Glechoma longituba significantly abolished these elevations in a dose-dependent manner. (Figure 1).

\section{Effect of Glechoma longituba on HK-2 cell proliferation and apoptosis}

$\mathrm{CaOx}$ treatment significantly decreased cell viability of HK-2 cells, but pre-treatment with Glechoma longituba significantly attenuated the effect of $\mathrm{CaOx}$ on HK-2 cells in a dosedependent manner (Figure 2 A). Moreover, Glechoma longituba pre-treatment reversed the elevated apoptosis induced by $\mathrm{CaOx}$ in $\mathrm{HK}-2$ in a dose-dependent manner (Figure 2 B). The western blotting results showed that $\mathrm{CaOx}$ induced the expression of Bax, cleaved-caspase3 and cleaved-caspase-9, and reduced the expression levels of Bcl-2, pro-caspase- 3 and 
A

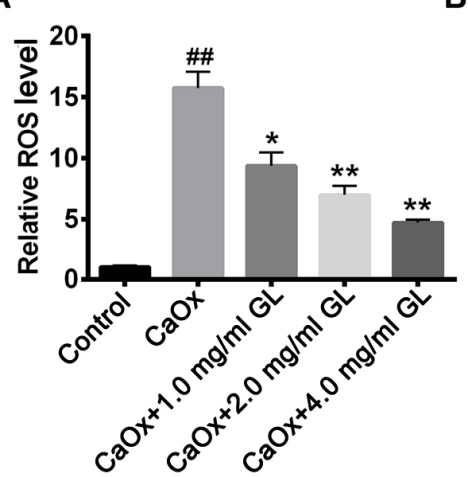

B

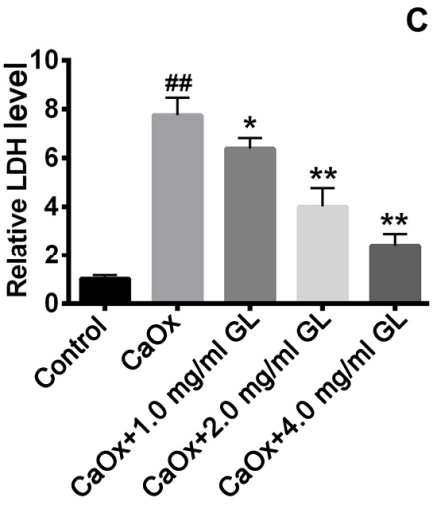

C

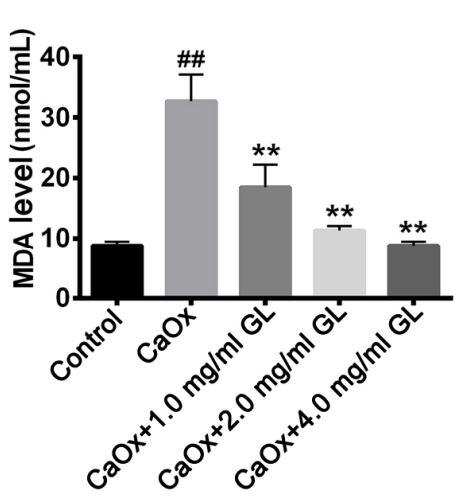

Figure 1: Expression levels of ROS, LDH and MDA in HK-2 cells treated with CaOx and Glechoma longituba. A, Relative ROS levels in HK-2 cells; B, relative LDH levels in HK-2 cells; and C, relative MDA levels in HK-2 cells. $\mathrm{ROS}$, reactive oxygen species; $\mathrm{LDH}$, lactate dehydrogenase; MDA, malondialdehyde; GL, Glechoma longituba. $\mathrm{CaOx}$, calcium oxalate. ${ }^{\#} p<0.01$, compared with the control group; $p<0.05,{ }^{* *} p<0.01$ compared with the $\mathrm{CaOx}$ group

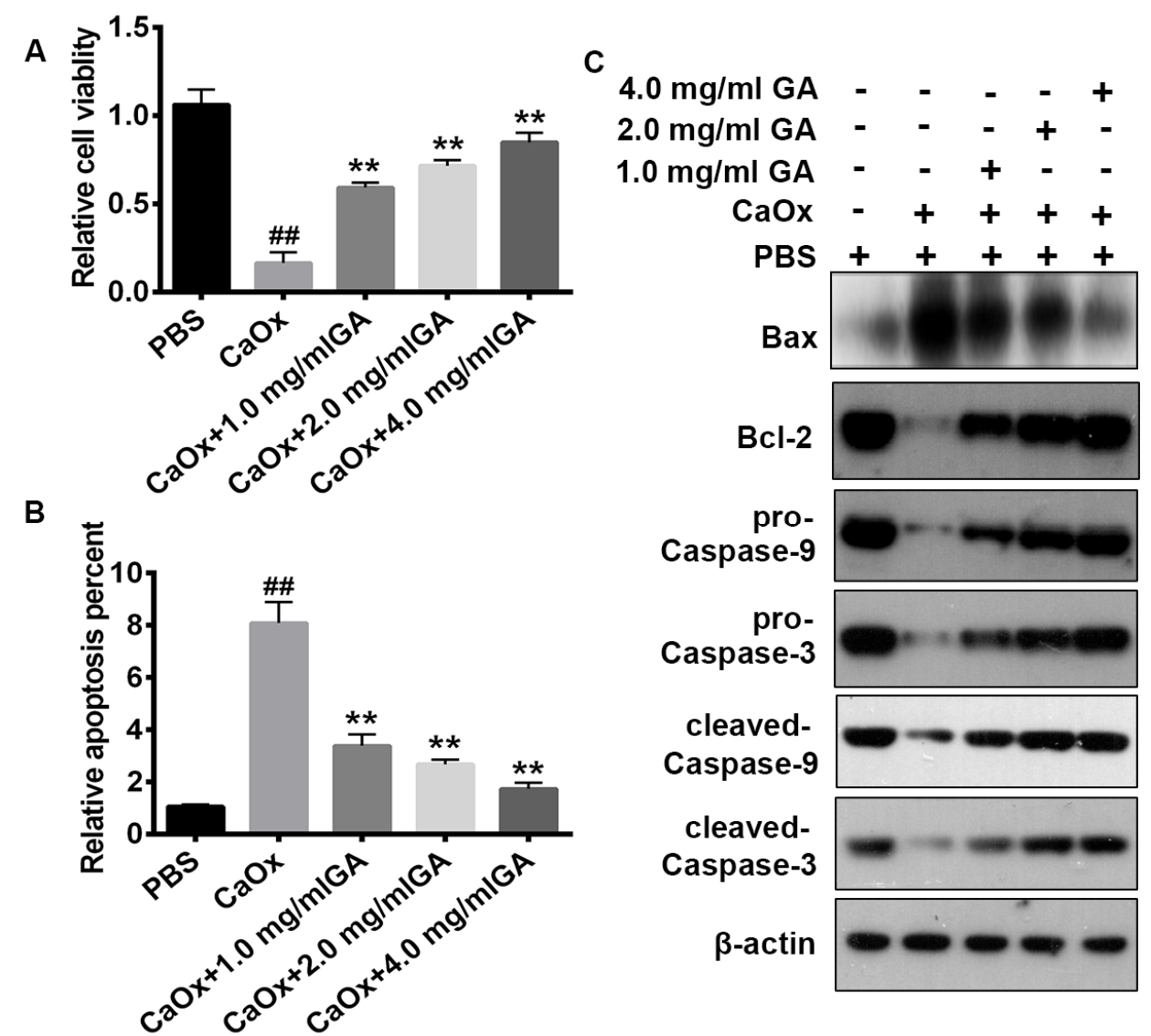

Figure 2: Glechoma longituba (GA) attenuated the effects of $\mathrm{CaOx}$ on the cell viability and inhibited apoptosis of HK-2. A, Cell viability of HK-2 cells was determined using the 3-(4,5-dimethylthiazol-2-yl)-2,5-diphenyltetrazolium bromide (MTT) assay; B, apoptosis of HK-2 cells was determined using flow cytometry; C, the expression of apoptosis-associated proteins in HK-2 cells was determined using western blotting. ${ }^{\# \#} p<0.01$ compared with the control group; $p<0.05,{ }^{* *} p<0.01$ compared with the CaOx group. CaOx, calcium oxalate; PBS, phosphatebuffered saline

pro-caspase-9 in HK-2 cells. However, Glechoma longituba pre-treatment reversed these effects in a dose-dependent manner (Figure $2 \mathrm{C}$ ).

\section{Effect of Glechoma longituba on the expression of Nrf2, HO-1 and NQO1}

The expression levels of Nrf2, HO-1 and NQO1 in HK-2 cells were determined using qRT-PCR. The analytical results showed that the expression 

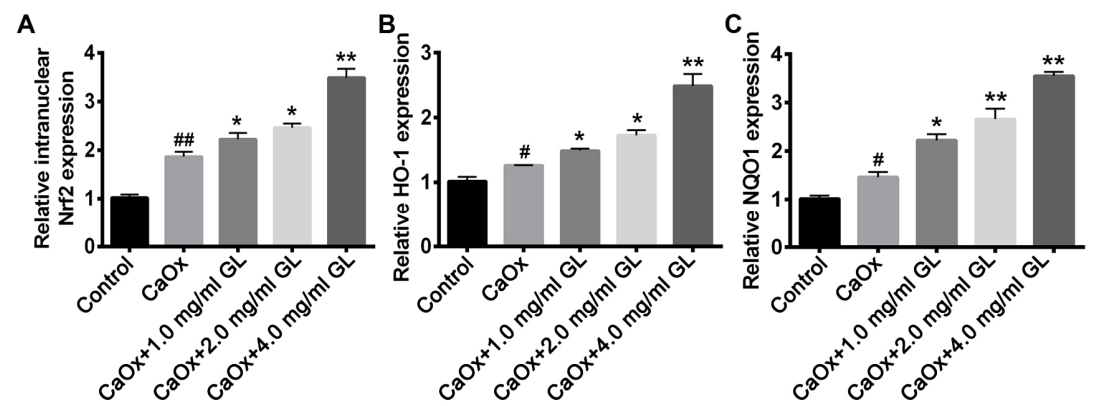

Figure 3: Relative expressions of the $\mathrm{Nrf}$, $\mathrm{HO}-1$ and $\mathrm{NQO} 1$ genes using quantitative real-time PCR. A, Relative expression of Nrf2; B, relative expression of HO-1; and C, relative expression of $N Q O 1 .{ }^{\# \#} p<0.01$ compared with the control group; $p<0.05,{ }^{* *} p<0.01$ compared with the $\mathrm{CaOx}$ group. $\mathrm{CaOx}$, calcium oxalate; GL, Glechoma longituba

A

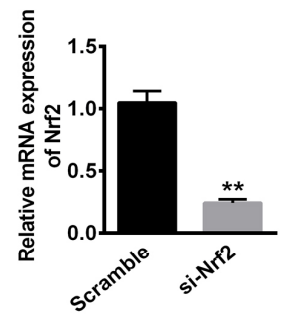

D

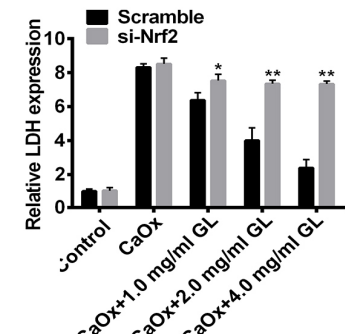

E

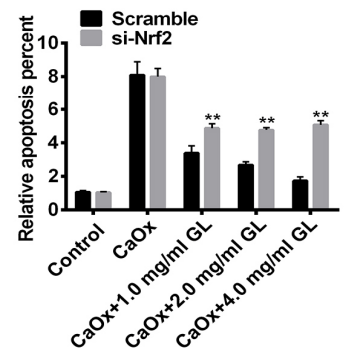

B

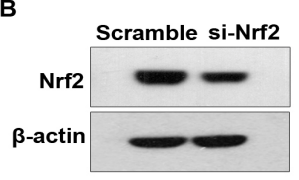

$\mathbf{F}$

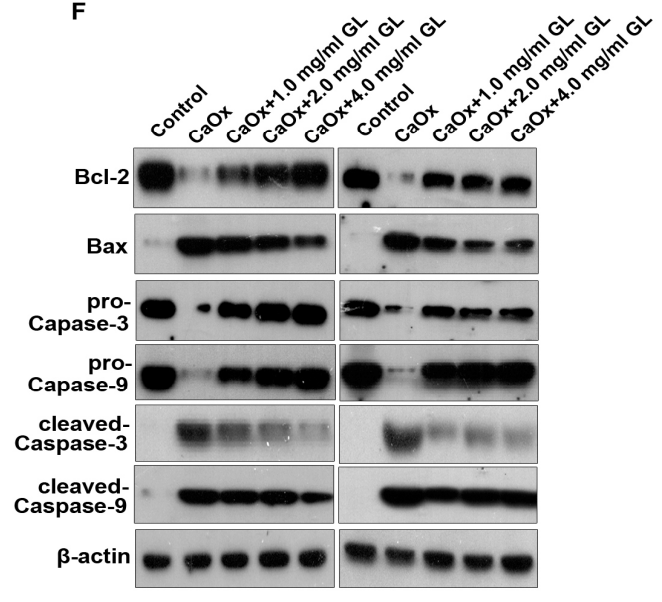

Figure 4: GL effects on si-Nrf2 in HK-2 cells attenuated the effects of GL against CaOx. A, Expression of the Nrf2 gene using quantitative real-time PCR in HK-2 cells; B, relative expression of Nrf2 protein as measured by western blotting in HK-2 cells; C, relative expression of reactive oxygen species (ROS) levels determined in HK-2 cells; $\mathrm{D}$, relative expression of lactate dehydrogenase (LDH) determined using a commercial kit in HK-2 cells; E, relative expression of apoptosis determined using flow cytometry in HK-2 cells; and F, expression levels of apoptosis-associated proteins using western blotting in HK-2 cells. ROS, reactive oxygen species; GL, Glechoma longituba; $\mathrm{CaOx}$, calcium oxalate. ${ }^{\star} p<0.05,{ }^{\star *} p<0.01$, compared with the scramble group

levels of Nrf2, $\mathrm{HO}-1$ and NQO1 were significantly upregulated following treatment with $\mathrm{CaOx}$, but Glechoma longituba pre-treatment markedly improved these effects in a dose-dependent manner (Figure 3).

\section{Knock-down Nrf2 abolished the effects of Glechoma longituba on cell apoptosis}

To further determine the underlying mechanism of Glechoma longituba, Nrf2 was knocked down in HK-2 cells, and the levels of ROS, LDH, and apoptosis were estimated after treating with $\mathrm{CaOx}$. The knockdown efficacy of $\mathrm{Nrf} 2$ was confirmed using both qRT-PCR (Figure $4 \mathrm{~A}$ ) and western blotting (Figure $4 \mathrm{~B}$ ). Compared with the control group, $\mathrm{CaOx}$ significantly increased the levels of ROS, LDH, and apoptosis in all groups, while Glechoma longituba pre-treatment abolished these elevations in all groups, but this effect was attenuated in the si-Nrf2 transfected group as compared with the scramble

Trop J Pharm Res, February 2018; 17(2): 229 
transfected group (Figure $4 \mathrm{C}-\mathrm{E}$ ). In addition, the western blotting also showed that Glechoma longituba pre-treatment did not reverse the increased expression of Bax-2, cleaved-caspase3 and cleaved-caspase-9, and the decreased expression of Bcl-2, pro-caspase-3 and caspase9 induced by $\mathrm{CaOx}$ in the si-Nrf2 HK-2 cells (Figure $4 \mathrm{~F}$ ).

\section{DISCUSSION}

Oxidative damage is considered an important promoter of renal epithelial damage and $\mathrm{CaOx}$ formation. In the current study, Glechoma longituba pre-treatment significantly abolished the elevations of ROS and apoptosis in HK-2 cells induced by $\mathrm{CaOx}$ via the $\mathrm{Nrf} / \mathrm{HO}-1$ signalling pathway. Glechoma longituba pretreatment also increased the viability of HK-2 cells after treatment with $\mathrm{CaOx}$. These findings indicated that Glechoma longituba had a promising effect on the alleviation of renal epithelial damage caused by $\mathrm{CaOx}$.

Glechoma longituba is a common plant distributed in Asia, America, and Europe. It has been widely used in disease treatment for centuries in China, including for dropsy, urolithiasis, asthma, and cholelithiasis [15]. The chemical constituents of this plant include phenolic acids, triterpenoids, essential oils, and flavonoids [16]. Among them, phenolic acids, such as caffeic, rosmarinic, chlorogenic acids, and flavonoids possess promising antioxidant properties [17-19]. Liang et al have shown that Glechoma longituba had anti-urolithic affects via inhibiting urinary stone formation and urolithiasisrelated protein expression levels, and elevating antioxidant levels [20].

Wang et al reported that Glechoma longituba decreased the oxidative, inflammatory and fibrotic levels via downregulating NF-KB, AP-1, and TGF- $\beta /$ Smad signalling pathway components in a bile duct ligation-induced liver injury model [21]. In the present study, Glechoma longituba pre-treatment significantly downregulated ROS, LDH and MDA levels induced by $\mathrm{CaOx}$. It also decreased the elevation of apoptosis, but increased the reduction of cell viability induced by $\mathrm{CaOx}$ in a dose-dependent manner. Thus, it was shown that $\mathrm{CaOx}$ treatment increased cell apoptosis via upregulating oxidative damage in renal cells, while Glechoma longituba possessed a relatively high antioxidant ability, which significantly reversed the elevated oxidative stress to suppress cell apoptosis and promote cell viability. These findings indicated that Glechoma longituba may provide a promising therapeutic method for nephrolithiasis treatment via decreasing cell oxidative stress.

Nrf2 is an oxidative stress responding transcriptional factor that regulates the expression of several antioxidant genes via binding to their antioxidant response elements [22]. Specifically, HO-1 and NQO1 are two important downstream targets of Nrf2 that respond to oxidative stimuli $[23,24]$. Liang et al have demonstrated that dihydroquercetin decreased oxidative stress by inducing the expression of HO-1 and NQO-1 via a Nrf2dependent antioxidant pathway [25]. Kang et al reported that Schisandrae semen essential oil attenuated oxidative damage via upregulating the $\mathrm{Nrf} 2 / \mathrm{HO}-1$ signalling pathway in $\mathrm{C} 2 \mathrm{C} 12$ murine skeletal muscle cells [26].

Sahu et al found that Lagerstroemia speciosa L. decreased apoptosis in isoproterenol-induced cardio-toxic mice via downregulating oxidative stress dependent on Nrf2/HO-1 [27]. $\mathrm{CaOx}$ is a common component of calculi in stone formers, and induces ROS to increase the oxidative damage in cells $[8,9]$. In the current study, we observed that $\mathrm{CaOx}$ treatment increased the expression of Nrf2, HO-1 and NQO-1, indicating that $\mathrm{CaOx}$ upregulated oxidative stress in $\mathrm{HK}-2$ cells, while HK-2 cells had the ability to respond to this stimulation via the Nrf-2 signalling pathway. A similar result was also reported by Lin et al in $\mathrm{H}_{2} \mathrm{O}_{2}$-treated $\mathrm{HK}-2$ cells [28]. However, Glechoma longituba pre-treatment promoted increased levels of Nrf2, HO-1 and NQO-1 in HK-2 cells, to prevent oxidative damage induced by $\mathrm{CaOx}$.

To further confirm this signal transduction mechanism, Nrf2 was knocked down in HK-2 cells and treated with Glechoma longituba pretreatment and/or $\mathrm{CaOx}$. The results showed that si-Nrf2 prevented the inhibitory effects of Glechoma longituba on the increased cell oxidative stress and apoptosis induced by $\mathrm{CaOx}$. These findings suggest that Glechoma longituba pre-treatment significantly attenuated cell oxidative damage and apoptosis induced by $\mathrm{CaOx}$ via Nrf2/HO-1 signalling pathway.

\section{CONCLUSION}

$\mathrm{CaOx}$ exerts oxidative stress on HK-2 cells, which significantly inhibits cell viability and increases cell apoptosis. Glechoma longituba has antioxidant effects that attenuate the cell oxidative damage and apoptosis induced by $\mathrm{CaOx}$ via the Nrf2/HO-1 signalling pathway, in a dose-dependent manner. These findings indicate that Glechoma longituba is a promising 
therapeutic agent for the treatment of nephrolithiasis.

\section{DECLARATIONS}

\section{Conflict of Interest}

No conflict of interest associated with this work.

\section{Contribution of Authors}

We declare that this work was done by the authors named in this article and all liabilities pertaining to claims relating to the content of this article will be borne by the authors.

\section{REFERENCES}

1. Wang H, Man L, Li G, Huang G, Liu N. Association between serum vitamin $D$ levels and the risk of kidney stone: evidence from a meta-analysis. Nutr J 2015; 15 : 1-5.

2. Jr SC, Smith AC, Hanley JM, Saigal CS. Prevalence of kidney stones in the United States. Eur Urol 2012; 62. 936-937.

3. Sas DJ, Hulsey TC, Shatat IF, Orak JK. Increasing incidence of kidney stones in children evaluated in the emergency department. J Pediatr 2010; 157: 132-137.

4. Tasian GE, Copelovitch L. Evaluation and medical management of kidney stones in children. J Urology 2014; 192: 1329-1336.

5. Antonelli JA, Maalouf NM, Pearle MS, Lotan Y. Use of the National Health and Nutrition Examination Survey to Calculate the Impact of Obesity and Diabetes on Cost and Prevalence of Urolithiasis in 2030. Eur Urol 2014; 66: 724.

6. Eisner BH, Goldfarb DS, Pareek G. Pharmacologic Treatment of Kidney Stone Disease. Urol Clin N Am 2013; 40: 21-30.

7. Mulay SR, Eberhard JN, Desai J, Marschner JA, Kumar SV, Weidenbusch M, Grigorescu M, Lech M, Eltrich N, Muller $L$, et al. Hyperoxaluria Requires TNF Receptors to Initiate Crystal Adhesion and Kidney Stone Disease. J AM Soc Nephrol 2016; 28: 761-768.

8. Baggio B, Gambaro G, Ossi E, Favaro S, Borsatti A. Increased urinary excretion of renal enzymes in idiopathic calcium oxalate nephrolithiasis. J Urol 1983; 129: 1161-1162.

9. Khan SR. Reactive oxygen species as the molecular modulators of calcium oxalate kidney stone formation: evidence from clinical and experimental investigations. J Urol 2013; 189:803-811.

10. Liang L, Li L, Tian J, Lee SO, Dang Q, Huang CK, Yeh S, Erturk E, Bushinsky D, Chang LS, et al. Androgen receptor enhances kidney stone-CaOx crystal formation via modulation of oxalate biosynthesis \& oxidative stress. Mol Endocrinol 2014; 28: 1291.
11. Ma MC, Chen YS, Huang HS. Erythrocyte Oxidative Stress in Patients with calcium oxalate stones correlates with stone size and renal tubular damage. Urol 2014; 83: 9-17.

12. Rodgers AL, Webber D, Ramsout R, Gohel MD. Herbal preparations affect the kinetic factors of calcium oxalate crystallization in synthetic urine: implications for kidney stone therapy. Urolithiasis 2014; 42: 221-225.

13. Gohel MDI, Wong SP. Chinese herbal medicines and their efficacy in treating renal stones. Urol Res 2006; 34 : 365-372.

14. Livak KJ, Schmittgen TD. Analysis of Relative Gene Expression Data Using Real-Time Quantitative PCR and the 2- $\Delta \Delta C$ T Method. Methods 2012; 25: 402-408.

15. Shan Q, Cao G, Cai H, Cai B. Simultaneous determination of four bioactive compounds in Glechoma longituba extracts by high performance liquid chromatography. Pharmacogn Mag 2013; 9: 216-219.

16. Yang NY, Duan JA, Li P, Qian SH. Chemical constituents of Glechoma longituba. APSB 2006; 41: 431-434.

17. Dorman HJD, KoşAr M, BaşEr KHC, Hiltunen R. Phenolic profile and antioxidant evaluation of Mentha $\times$ piperita $L$. (peppermint) extracts. Nat Prod Commun 2009; 4: 535 542.

18. Zaabat N, Hay AE, Michalet S, Darbour N, Bayet C, Skandrani $I$, et al. Antioxidant and antigenotoxic properties of compounds isolated from Marrubium deserti de Noé. Food Chem Toxicol 2011; 49:33283335.

19. Döring AS, Petersen M. Production of caffeic, chlorogenic and rosmarinic acids in plants and suspension cultures of Glechoma hederacea. Phytochem Lett 2014; 10: cxicxvii.

20. Liang $Q$, Li X, Zhou W, Su Y, He S, Cheng S, Lu JZ, Cao $W J$, Yan YK, Pei $X X$, et al. An Explanation of the underlying mechanisms for the in vitro and in vivo antiurolithic activity of Glechoma longituba. Oxid Med Cell Longev 2016; 2016:1-11.

21. Wang $Y Y$, Lin SY, Chen WY, Liao SL, Wu CC, Pan PH, Chou ST, and Chen CJ. Glechoma hederacea extracts attenuate cholestatic liver injury in a bile duct-ligated rat model. J Ethnopharmcol 2017; 204:58.

22. Niture SK. Regulation of Nrf2-an update. Free Radical Bio Med 2014; 66:36-44.

23. Li L, Dong H, Song E, Xu X, Liu L, Song Y. Nrf2/ARE pathway activation, $\mathrm{HO}-1$ and NQO1 induction by polychlorinated biphenyl quinone is associated with reactive oxygen species and PI3K/AKT signaling. Chem Biol Interact 2014; 209:56-67.

24. Loboda A, Damulewicz M, Pyza E, Jozkowicz A, Dulak J. Role of Nrf2/HO-1 system in development, oxidative stress response and diseases: an evolutionarily conserved mechanism. Cell Mol Life Sci 2016; 73:32213247.

25. Liang L, Gao C, Luo M, Wang W, Zhao C, Zu Y, Efferth $T$, and $\mathrm{Fu} Y \mathrm{YJ}$. Dihydroquercetin (DHQ) Induced HO-1 and NQO1 Expression against Oxidative Stress through 
the Nrf2-Dependent Antioxidant Pathway. J Agr Food Chem 2013; 61:2755-2761.

26. Kang JS, Han MH, Kim GY, Kim CM, Chung HY, Hwang HJ, Kim BW, Choi YH. Schisandrae semen essential oil attenuates oxidative stress-induced cell damage in C2C12 murine skeletal muscle cells through Nrf2amediated upregulation of HO-1. Int $\mathrm{J} \mathrm{Mol} \mathrm{Med}$ 2015; 35:453-459.

27. Sahu BD, Kuncha $M$, Rachamalla SS, Sistla $R$. Lagerstroemia speciosa $L$. attenuates apoptosis in isoproterenol-induced cardiotoxic mice by inhibiting oxidative stress: possible role of Nrf2/HO-1. Cardiovasc Toxicol 2015; 15:10-22.

28. Lin M, Li L, Zhang $Y$, Zheng L, Xu M, Rong R, Zhu TY. Baicalin ameliorates $\mathrm{H}_{2} \mathrm{O}_{2}$ induced cytotoxicity in $\mathrm{HK}-2$ cells through the inhibition of ER stress and the activation of Nrf2 signaling. Int J Mol Sci 2014; 15: 12507-12522. 